\title{
Section of Geriatric Medicine of the Medical Society of Göteborg, Sweden
}

(Meeting, October 7, 1959)

Symposium on Cerebrovascular Disease

1. Dr. S.Eckerström (Göteborg). General aspects

The characteristics of the cerebral circulation, with the arterial pressure as the most important factor regulating the cerebral blood flow, were described. The importance of recognizing early signs and symptoms of transient ischaemia of the brain caused by obstruction or narrowing of a cerebral artery was pointed out. The problems of differential diagnosis of conditions that may simulate the effects of cerebral thrombosis or intra-cerebral haemorrhage were discussed and especially the need for making the early diagnosis of obstruction of the internal carotid artery in the neck by angiography. The development of anticoagulant therapy makes the differential diagnosis of thrombosis from haemorrage of a very practical importance.

The factors of significance which accelerate the development of atherosclerosis such as hypertension, hypercholesterolaemia, diabetes, polycythaemia, thrombocythae-mia and the important therepeutic emphasis on anticoagulants, diet restrictions and fibrinolytic agents were discussed. Anticoagulant therapy is successful in preventing transient ischaemic attacks in early cases of cerebral thrombosis.

2. Dr. T. Dalsgaard-Nielsen (Copenhagen). Diagnosis and therapy of acute cerebrovascular diseases

Dr. Nielsen gave a review of the problem based upon his personal experiences from about 2500 cases of acute cerebral catastrophes referred to the neurological department of Fredriksberg Hospital, Copenhagen during the last 15 years. The first object of diagnosis is to find out in what cases thrombosis, haemorrhage or embolism cause the symptoms or signs of stroke and in what cases there are post-traumatic vascular rupture, cerebral tumour, intracranial metastases, mental confusion caused by acute febrile conditions, or intoxications, etc.

During the last 15 years better diagnostic methods make it feasible to carry out new forms of therapy. These are:

1. Out of bed as early as possible (lst-3rd day). 2. Physiotherapy starting during the first week. 3 . Thorough care of mouth and skin. 4. Adequate supply of fluid and food. 5. Vasodilators (e.g. nicotinic acid, nitroglycerine, theobromine). 6. Dehydration if necessary. 7. Parenteral supply of glucose or sodium bicarbonate. 8. Oxygen inhalation or antibiotics if necessary. 9. Urinary catheter for urinary incontinence. 10. Stimulation. 11. Care of the underlying disease. 12.

Neurosurgery. 13. Orthopaedic care. 14. Anticoagulant therapy against complicating thrombosis and embolism. 15. Speech therapy for aphasia.

Since the introduction of these active diagnostic and therapeutic methods the results improved a little, but not until recently, with the attainment of more accurate diagnosis, by starting a general examination with lumbar puncture in all cases and E.E.G. and angiography was it possible to show that about $75 \%$ of the cerebral vascular accidents were due to an occlusion (thrombosis) of 
a cerebral artery and about $20 \%$ to an intracerebral haemorrhage. It is now possible to make extensive use of anti-coagulant therapy and so the thrombo-embolic complications have practically disappeared.

In the following discussion Dr. /. Wickbom spoke of the neuro-radiological aspects of the problem, Dr. L.Hannsson on aspects of the surgical treatment of stenosis and thrombosis of the internal carotid, vertebral and common carotid arteries and Dr. S. Lindgren experience from the University clinic of neurosurgery. 